\title{
Uniqueness of the AdS spacetime among static vacua with prescribed null infinity
}

\author{
Oussama Hijazi and Sebastián Montiel ${ }^{1}$
}

\begin{abstract}
We prove that an $(n+1)$-dimensional spin static vacuum with negative cosmological constant whose null infinity has a boundary admitting a non-trivial Killing spinor field is the AdS spacetime. As a consequence, we generalize previous uniqueness results by $\mathrm{X}$. Wang [40, 42] and by Chruściel-Herzlich [20] and introduce, for this class of spin static vacua, some Lorentzian manifolds which are prohibited as null infinities.
\end{abstract}

\section{Introduction}

An $(n+1)$-dimensional static vacuum spacetime with negative cosmological constant $\Lambda=-n$ is determined by a triple $(M, g, V)$, where $(M, g)$ is an $n$-dimensional connected Riemannian manifold standing for the unchanging slices of constant time and $V \in C^{\infty}(M)$ is a positive smooth function on $M$ such that

$$
\operatorname{Ric}_{g}+n g=\frac{\nabla^{2} V}{V}, \quad \Delta V=n V
$$

where Ric $_{g}$ is the Ricci tensor, $\nabla^{2}$ is the Hessian operator and $\Delta=\operatorname{trace} \nabla^{2}$ is the Laplacian of the Riemannian manifold $(M, g)$. Taking traces in the first of these two equations and taking into account the second one, we conclude immediately that

$$
R_{g}=-n(n-1)
$$

where $R_{g}$ is the scalar curvature of $(M, g)$.

The paradigmatic example of a static vacuum is given by choosing $(M, g)=\left(\mathbb{H}^{n}, g_{\mathbb{H}}\right)$ the hyperbolic space of constant sectional curvature -1 ,

\footnotetext{
${ }^{1}$ The second author was partially supported by a Spanish MEC-FEDER grants Nos. MTM2007-61775 and MTM2011-22547
} 
which can be viewed as the upper sheet of a hyperquadric in the Minkowski spacetime $\left(\mathbb{R}_{1}^{n+1},\langle\rangle,\right)$, namely,

$$
\mathbb{H}^{n}=\left\{\left.p \in \mathbb{R}_{1}^{n+1}|| p\right|^{2}=-1, p_{0}>0\right\} .
$$

This is a spacelike hypersurface and the metric $g_{\mathbb{H}}$ induced on $\mathbb{H}^{n}$ from $\langle$, is a Riemannian metric making $\left(\mathbb{H}^{n}, g_{\mathbb{H}}\right)$ a space form of sectional curvature -1 . In particular, $\operatorname{Ric}_{g_{\mathbb{H}}}=-(n-1) g_{\mathbb{H}}$. In this representation of the hyperbolic space, it is easy to see that, for each fixed $a \in \mathbb{R}_{1}^{n+1}$, the height function $h_{a}: \mathbb{H}^{n} \rightarrow \mathbb{R}$ given by

$$
h_{a}(p)=\langle p, a\rangle, \quad \forall p \in \mathbb{H}^{n}
$$

satisfies the Obata type equation (see [33])

$$
\nabla^{2} h_{a}=h_{a} g_{\mathbb{H}} \cdot
$$

As a consequence, $\Delta h_{a}=n h_{a}$. Moreover, when $a$ is chosen to be lightlike or timelike vector with $a_{0}<0$, we have $h_{a}>0$. Then the triple $\left(\mathbb{H}^{n}, g_{\mathbb{H}}, h_{a}\right)$ is a static vacuum for such causal $a \in \mathbb{R}_{1}^{n+1}$. Indeed, the static vacuum determined by this triple is nothing but the Anti de Sitter (AdS) spacetime.

However, there is a subtle difference between the case where $a$ is timelike and the case where $a$ is lightlike. In the former, the level hypersurfaces of $h_{a}$ are umbilical round hyperspheres in $\mathbb{H}^{n}$ allowing to write the hyperbolic metric $g_{\mathbb{H}}$ as a warped product metric and describe the function $h_{a}$ as follows:

$$
g_{\mathbb{H}}=d r^{2}+\sinh ^{2} r\langle,\rangle_{\mathbb{S}^{n-1}}, \quad h_{a}=\cosh r, \quad r \in[0,+\infty[.
$$

Consequently, the Lorentzian metric of AdS can be written as follows

$$
g_{A d S}=-\cosh ^{2} r d t^{2}+d r^{2}+\sinh ^{2} r\langle,\rangle_{\mathbb{S}^{n-1}}, \quad t \in \mathbb{R}, r \in[0,+\infty[.
$$

This description shows that it is conformal to a cylindrical Lorentzian metric. In fact, we have

$$
g_{A d S}=\cosh ^{2} r\left(-d t^{2}+d s^{2}+\sin ^{2} s\langle,\rangle_{\mathbb{S}^{n-1}}\right),
$$

where $s=-\frac{\pi}{2}+2 \arctan e^{r} \in\left[0, \frac{\pi}{2}[\right.$, that is,

$$
g_{A d S}=\cosh ^{2} r\left(-d t^{2}+g_{\mathbb{S}_{+}^{n}}\right),
$$

where $g_{\mathbb{S}_{+}^{n}}$ is the unit round metric on the open hemisphere $\mathbb{S}_{+}^{n}$. From this conformal equivalence between Lorentzian metrics, it is straightforward to 
see that the conformal null infinity of the AdS spacetime is nothing but the cylinder $\mathbb{R} \times \mathbb{S}^{n-1}=\mathbb{R} \times \partial \mathbb{S}_{+}^{n-1}$ with the product Lorentzian structure $-d t^{2}+g_{\mathbb{S}^{n-1}}$.

In the second case, the level hypersurfaces of $h_{a}$ are umbilical flat Euclidean spaces (horospheres) in $\mathbb{H}^{n}$. The corresponding foliation also allows to write the hyperbolic metric $g_{\mathbb{H}}$ as another warped product and describe in a corresponding manner the function $h_{a}$ :

$$
\left.g_{\mathbb{H}}=d r^{2}+e^{2 r}\langle,\rangle_{\mathbb{R}^{n-1}}, \quad h_{a}=e^{r}, \quad r \in\right] 0,+\infty[.
$$

Consequently we obtain another expression for the AdS metric:

$$
\left.g_{A d S}=-e^{2 r} d t^{2}+d r^{2}+e^{2 r}\langle,\rangle_{\mathbb{R}^{n-1}}, \quad t \in \mathbb{R}, r \in\right] 0,+\infty[.
$$

In this case, the height function $h_{a}$ provides a different conformal equivalence for the AdS metric. Indeed, we have

$$
g_{A d S}=e^{2 r}\left(-d t^{2}+d s^{2}+s^{2}\langle,\rangle_{\mathbb{R}^{n-1}}\right),
$$

where $s=e^{-r} \in[0,1[$, that is,

$$
g_{A d S}=e^{2 r}\left(-d t^{2}+g_{B^{n}}\right)
$$

where $B^{n}$ is the open unit disc of the Euclidean space $\mathbb{R}^{n}$ and $g_{B^{n}}$ the flat metric. As in the first case, from this conformal equivalence for $g_{A d S}$, it is straightforward to see that the conformal null infinity of the AdS spacetime is viewed again as $\mathbb{R} \times \mathbb{S}^{n-1}=\mathbb{R} \times \partial B^{n}$ with its standard cylindrical Lorentzian structure $-d t^{2}+g_{\mathbb{S}^{n-1}}$.

Then, it is clear that, in the particular case of the AdS spacetime $(M, g, V)$ $=\left(\mathbb{H}^{n}, g_{\mathbb{H}}, h_{a}\right)$, the positive function $V^{-1}=h_{a}^{-1}$ is also a defining function for the hyperbolic space $\mathbb{H}^{n}$, which is the prototypical example of a conformally compact Riemannian manifold. This simply means that the function $V^{-1}$ and the Riemannian metric $\bar{g}=V^{-2} g=h_{a}^{-2} g_{\mathbb{H}}$ extends to an $(n+1)$ dimensional manifold with boundary $\bar{M}\left(\mathbb{S}_{+}^{n}\right.$ or $\left.B^{n}\right)$ whose interior is diffeomorphic to $\mathbb{H}^{n}$ (for definitions, generalities and main facts on conformally compact Riemmanian manifolds, see [3-5, 13]).

Considering the above facts, we will say that a static vacuum $(M, g, V)$ is asymptotically locally anti de Sitter (ALAdS, in short) if $(M, g)$ is a conformally compact Riemannian manifold and the positive smooth function $V^{-1}$ is a defining function, that is, if there exists an $(n+1)$-dimensional manifold with boundary $\bar{M}$ such that $M$ is its interior and the function $V^{-1}$ 
extends smoothly to $\bar{M}$ in such a way that $V^{-1}{ }_{\mid \partial M}=0,\left(d V^{-1}\right)_{\mid \partial M} \neq 0$, and the metric $\bar{g}=V^{-2} g$ extends to $\bar{M}$ as well (indeed, in order to obtain the results below it would suffice to have a weaker regularity for $\bar{g}$ ). Hence, the extended metric $\bar{g}$ induces a Riemannian metric $\gamma=\bar{g}_{\mid \partial M}$ on the boundary at infinity $\partial M=\partial \bar{M}$ of $(M, g)$. Note that, even if $M$ is assumed to be connected, its boundary at infinity could be disconnected. Moreover, the Riemannian manifold $\left(\partial M, V^{-2} g_{\mid \partial M}\right)$ is the Riemannian slice of the conformal null infinity $\mathcal{J}=\left(\mathbb{R} \times \partial M,-d t^{2}+\gamma\right)$ of the spacetime determined by $(M, g, V)$.

In general, a conformally compact Riemannian manifold is asymptotically negatively curved. But if it has also asymptotically constant scalar curvature, say $-n(n-1)$ after a suitable normalization, that is, if $R_{g} \rightarrow$ $-n(n-1)$ when one approaches $\partial M$, then the manifold is asymptotically locally (weakly, according to [41]) hyperbolic (ALH in short). For this class of manifolds we have $K_{g} \rightarrow-1$ at infinity. As a consequence, the Ricci tensor satisfies $\operatorname{Ric}_{g} \rightarrow-(n-1) g$, that is, the manifold seems to be Einstein with Ricci curvature $-(n-1)$ when one moves towards infinity. In the case of a static vacuum $(M, g, V)$, from $(2)$, we know that $(M, g)$ has constant scalar curvature $R_{g}=-n(n-1)$. Thus, the spatial slice of an ALAdS static vacuum is an ALH Riemannian manifold. When the conformal infinity $\partial M$ of $(M, g)$ is an $(n-1)$-dimensional hypersphere, we will say that it is asymptotically anti de Sitter (AAdS in short). It is obvious that, in this case, $(M, g)$ is an asymptotically hyperbolic (AH in short) Riemannian manifold. In this situation, P. T. Chruściel and M. Herzlich (for $n=3$ ) and X. Wang (for any $n$ ) generalized a former result by W. Boucher, G. Gibbons and G. Horowitz ([21, Theorem 4.5], [42, Theorem 1], cfr. [15]) by proving the following uniqueness result :

Let $(M, g, V)$ be an $(n+1)$-dimensional AAdS static vacuum. Suppose that $M$ is a spin manifold and that its boundary at infinity $(\partial M, \gamma)$ is a round $(n-1)$-dimensional sphere. Then $(M, g, V)$ is the AdS spacetime.

We will strengthen this result in two directions. First, we will allow the ALAdS static vacuum $(M, g, V)$ to have a non-spherical boundary at infinity $\partial M$ and we will not require the metric $\gamma$ to have constant sectional curvature. Using the spin setting, our strategy will be to slightly modify $V$, if necessary, in order to get a compactification of $(M, g)$ with non-negative scalar curvature and constant inner mean curvature and then apply, to the corresponding induced metric on $\partial M$, a previous estimate by $\mathrm{X}$. Zhang and 
ourselves (see [30, 31]) for the spectrum of the Dirac operator on spin Riemannian manifolds which bound compact domains. Indeed, the goal of this paper is to prove the following result and to analyse some of its consequences (see Theorem 7 below).

Let $(M, g, V)$ be an $(n+1)$-dimensional ALAdS static vacuum. Suppose that $M$ is a spin manifold.

1) If the boundary at infinity $(\partial M, \gamma)$ admits a non-trivial Killing, then $(M, g, V)$ is the AdS spacetime.

2) The boundary at infinity $(\partial M, \gamma)$ cannot admit a non-trivial parallel spinor.

We emphasize that the spin structure considered on $\partial M$ is just the induced one from that of $M$.

Note that, if $\partial M$ is spherical, then $\partial M$ admits a unique spin structure and if $\gamma$ is the unit round metric, then the Riemannian spin manifold $(\partial M, \gamma)$ supports a maximal number of independent Killing spinor fields (see [11, p.37], [25, Examples A.1.3.2]). Then the rigidity result by X. Wang and P. Chruściel and M. Herzlich is a direct consequence of 1) in our result quoted above (see Theorem 7). It is a well-known fact that there are compact non-spherical spin Riemannian manifolds carrying non-trivial Killing and parallel spinors (see [7] and [38, 39]). Thus, Theorem 7 implies the nonexistence of ALAdS spin static vacua with these types of spatial infinities. In particular, it is well-known that flat tori admit non-trivial parallel spinors. So, they cannot appear as the boundary at infinity $(\partial M, \gamma)$ of a ALAdS static vacuum. This particular case of 2 ) in our main result was also obtained by $\mathrm{X}$. Wang in [40, Theorem 6.1].

\section{Special conformal compactifications of spatial slices}

We already observed that the spatial slices $(M, g)$ of an ALAdS static vacuum $(M, g, V)$ are ALH manifolds. This geometrical feature reflects an equivalent analytical property which is satisfied by all the defining functions $\rho$ of $(M, g)$, namely, $|\bar{\nabla} \rho|_{\mid \partial M}=1$, where the gradient and the length are computed with respect to the same extended metric $\bar{g}=\rho^{2} g$ (see, for instance, $[12$, p. 59] or $[5$, Appendix]) corresponding to the defining function $\rho$ itself. This means that the vector field $\bar{\nabla} \rho=\rho^{-2} \nabla \rho$, restricted to $\partial M$, is an inner unit field normal to $\partial M$ with respect to the extended metric $\bar{g}$. In 
particular, since $V^{-1}$ is a defining function for $(M, g)$, we have that

$$
\bar{\nabla} V_{\mid \partial M}^{-1}=-\nabla V_{\mid \partial M} \perp T \partial M, \quad\left|\bar{\nabla} V^{-1}\right|_{\mid \partial M}=\left.\frac{|\nabla V|}{V}\right|_{\partial M}=1,
$$

where the last equality is to be understood as a limit. Also, for any other defining function $\rho$ defined on $\bar{M}$, we have

$$
1=\left.\frac{|\nabla V|}{V}\right|_{\partial M}=\left.\frac{|\nabla \rho|}{\rho}\right|_{\partial M}, \quad 0<(\rho V)_{\mid \partial M}<+\infty,
$$

where the last equality holds since the two vector fields $\nabla V$ and $\rho^{-2} \nabla \rho$ are parallel along $\partial M$.

In the general case, when the ALH manifold $(M, g)$ is not coming from a static vacuum, and hence it has not necessarily constant scalar curvature, if we assume that it is conformally compact of class at least $C^{3, \alpha}$, we may modify any defining function in a suitable way to obtain the so called geodesic defining functions.

Lemma 1. [4, Section 3], [2, Lemma 5.4], [26, Lemma 5.2], [35, Lemma 5.1], [41, Lemma 2.2] Let $(M, g)$ be an ALH manifold of class $C^{m, \alpha}, m \geq 3$. For each choice of a metric $\gamma$ on its conformal infinity $\partial M$, there exists a unique defining function $r \in C^{m-1, \alpha}(\bar{M})$ such that the extended conformal metric $\widehat{g}=r^{2} g$ is of class $C^{m-1, \alpha}, \gamma=\widehat{g}_{\mid \partial M}$ and $|\widehat{\nabla} r| \equiv 1$ in a collar neighbourhood of $\partial M$.

The existence of geodesic defining functions on conformally compact ALH manifolds is a key point to obtain many of their main features. In particular, it is possible to prove the connectedness of its boundary when one has both a lower bound on the Ricci tensor and a decay condition at infinity on the scalar curvature, along with the non-negativity of the conformal structure at the infinity. This connectedness was proved by E. Witten and S.-T. Yau for positive conformal infinities and M. Cai and G. Galloway for null conformal infinities ([18, 43], [3, Theorem 4.1], cf. also [29, Theorem 2]). When the given ALH manifold $(M, g)$ is the spatial slice of a static vacuum $(M, g, V)$, on one hand, we know from (2) that the scalar curvature is not only asymptotically decaying to $-n(n-1)$, but it is in fact exactly $-n(n-1)$. However, on the other hand, we have no lower bounds for the Ricci tensor. So the aforementioned connectedness results do not directly apply to our situation. Yet, it is possible to use the standard trick to finally obtain a similar conclusion as in the Riemannian case. In 
fact, it suffices to consider the Riemannian metric $\tilde{g}=V^{2} d \theta^{2}+g$ defined on $\mathbb{S}^{1} \times M$, which is an Einstein metric (see [36]) with conformal infinity $\left(\mathbb{S}^{1} \times \partial M,\left[d \theta^{2}+V^{-2} g_{\mid \partial M}\right]\right)$. Then, we can apply the above connectedness results and conclude that $\mathbb{S}^{1} \times \partial M$ must be connected. So the same conclusion is valid for $\partial M$. We summarize it in the following proposition.

Proposition 2. Let $(M, g, V)$ be an ALAdS static vacuum such that the boundary at infinity $(\partial M, \gamma)$ of its spatial slices $(M, g)$ has non-negative scalar curvature. Then $\partial M$ is connected.

On the other hand, in order to study the continuous spectrum of the Laplacian on an ALH Einstein manifold $(M, g)$, J. Lee proved in [35] the existence of positive solutions of the eigenvalue equation $\Delta u=(n+1) u$ and studied their growth at infinity $\partial M$. Some years later, J. Qing used in [37] these particular eigenfunctions as defining functions to partially compactify $(M, g)$ and gave a beautiful proof, based on the positive mass theorem, of the rigidity of the hyperbolic space among all the ALH Einstein manifolds whose prescribed boundary at infinity is a round sphere. In fact, he aimed to drop the spin assumption in the corresponding result by L. Andersson and $\mathrm{M}$. Dahl $([2])$. In our case, $(M, g)$ is the spatial slice of a static vacuum $(M, g, V)$ and thus it is an ALH manifold with constant scalar curvature, although not necessarily Einstein. However, we have a positive solution of the eigenvalue problem studied by J. Lee for the Laplace operator $\Delta$ of $g$, namely, the function $V$. Then we adapt the results in [35, Section 5] in order to get, by Lemma 1, a sufficiently good description of the asymptotical behaviour of $V$ in terms of the geodesic defining function on $(M, g)$ associated with $\gamma$. For this, we need to prove the following two lemmae.

Lemma 3. Let $(M, g, V)$ be an $A L A d S$ static vacuum and $\varepsilon \geq 0$ a real number. Then the function $(V+\varepsilon)^{-1}$ is a defining function for the ALH manifold $(M, g)$ such that the corresponding extended Riemannian metric $g^{*}=(V+\varepsilon)^{-2} g$ has scalar curvature

$$
R_{g^{*}}=n(n-1)\left(V^{2}-|\nabla V|^{2}-\varepsilon^{2}\right) .
$$

In particular, the function $V^{2}-|\nabla V|^{2}$ extends to the compact manifold with boundary $\bar{M}$.

Proof. Since $V^{-1}$ is a positive smooth function on $M$ extendable to $\bar{M}$ and vanishing on $\partial M$, we have that $(V+\varepsilon)^{-1}=V^{-1} /\left(1+\varepsilon V^{-1}\right)$ is another positive smooth function on $M$ extendable to $\bar{M}$ and vanishing on $\partial M$ as well. 
It is immediate to see that

$$
\left(d(V+\varepsilon)^{-1}\right)_{\mid \partial M}=\left(d V^{-1}\right)_{\mid \partial M} \neq 0 .
$$

Moreover the metric

$$
g^{*}=(V+\varepsilon)^{-2} g=\left(\frac{1}{1+\varepsilon V^{-1}}\right)^{2} V^{-2} g
$$

defined on $M$ extends to a metric on $\bar{M}$ with the same regularity as that of the metric $V^{-2} g$. So, $(V+\varepsilon)^{-1}$ is a defining function for $(M, g)$. Moreover, this metric $g^{*}$, restricted to $\partial M$, gives

$$
g^{*}{ }_{\mid \partial M}=\left((V+\varepsilon)^{-2} g\right)_{\mid \partial M}=\left(V^{-2} g\right)_{\mid \partial M} .
$$

In particular, $\left(M^{*}, g^{*}\right)=\left(\bar{M},(V+\varepsilon)^{-2} g\right)$ is a conformal compactification of the ALH manifold $(M, g)$. Now, rewrite the conformal change between the metrics $g$ and $g^{*}$ as

$$
g=(V+\varepsilon)^{2} g^{*},
$$

then the relation between the associated Ricci tensors $\mathrm{Ric}_{g^{*}}$ and $\mathrm{Ric}_{g}$ on the open manifold $M$ (see [12, p. 59] or [5, Appendix]), is given by

$$
\operatorname{Ric}_{g^{*}}=\operatorname{Ric}_{g}+(n-2) \frac{\nabla^{2} V}{V+\varepsilon}+\frac{\Delta V}{V+\varepsilon} g-(n-1) \frac{|\nabla V|^{2}}{(V+\varepsilon)^{2}} g .
$$

Taking traces with respect to $g$ and multiplying by $(V+\varepsilon)^{2}$, one gets for the corresponding scalar curvatures

$$
R_{g^{*}}=(V+\varepsilon)^{2} R_{g}+2(n-1)(V+\varepsilon) \Delta V-n(n-1)|\nabla V|^{2} .
$$

As we pointed out in (1) and (2), since $R_{g}=-n(n-1)$ and the function $V$ is an eigenfunction of $\Delta$ associated with the eigenvalue $-n$, we finally get (4).

An important consequence of Lemma 3 is the following information about the asymptotical behaviour of the Ricci tensor of the spatial slice $(M, g)$ when one approaches to $\partial M$ along the direction $\nabla V$.

Lemma 4. Let $\rho$ be any defining function of the ALH spatial slice $(M, g)$ of an ALAdS static vacuum. Then

$$
\lim _{\rho \rightarrow 0} \frac{1}{\rho^{2}}\left(\operatorname{Ric}_{g}+(n-1) g\right)\left(\frac{\nabla \rho}{\rho}, \frac{\nabla \rho}{\rho}\right)=0 .
$$


Proof. From Lemma 3, we know that $V^{2}-|\nabla V|^{2}$ is a differentiable function on the compact manifold $\bar{M}$. Let $\bar{N}$ be the inner unit vector field normal to $\partial M$ with respect to the extended metric $\bar{g}=V^{-2} g$. Then, the Lee derivative of the function $V^{2}-|\nabla V|^{2}$ in the direction of the vector field $\frac{1}{2} \bar{N}$, is given by

$$
\frac{1}{2} \bar{N} \cdot\left(V^{2}-|\nabla V|^{2}\right)=\left(V g(\nabla V, \bar{N})-\left(\nabla^{2} V\right)(\bar{N}, \nabla V)\right)_{\mid \partial M} \cdot
$$

But, by (3) and previous comments, we have $\bar{N}=-\nabla V_{\mid \partial M}$. Thus

$$
\frac{1}{2} \bar{N} \cdot\left(V^{2}-|\nabla V|^{2}\right)=\left(\left(\nabla^{2} V\right)(\nabla V, \nabla V)-V|\nabla V|^{2}\right)_{\mid \partial M} \cdot
$$

Now, from the first equality in (1), it follows

$$
\frac{1}{2} \bar{N} \cdot\left(V^{2}-|\nabla V|^{2}\right)=\left(V\left(\operatorname{Ric}_{g}+(n-1) g\right)(\nabla V, \nabla V)\right)_{\mid \partial M} .
$$

Using again (3), we know that

$$
\nabla V_{\mid \partial M}=\left.\frac{\nabla \rho}{\rho^{2}}\right|_{\partial M}, \quad V \rho=O(1),
$$

hence the existence of the following limit

$$
\lim _{\rho \rightarrow 0} \frac{1}{\rho^{3}}\left(\operatorname{Ric}_{g}+(n-1) g\right)\left(\frac{\nabla \rho}{\rho}, \frac{\nabla \rho}{\rho}\right),
$$

which implies (6).

Now, we dispose of all the necessary tools to control the asymptotic behavior of the function $V$ in the given static vacuum $(M, g, V)$.

Proposition 5. (Cfr. [35, Proposition 4.1, Lemmas 5.1 and 5.2] and [27, Lemma 2.1]) Let $(M, g, V)$ be an $(n+1)$-dimensional, $n \geq 3$, ALAdS static vacuum and $r$ the geodesic defining function associated with the metric $\gamma=$ $V^{-2} g_{\mid \partial M}$ on the boundary at infinity $\partial M$ of its spatial slices, according to Lemma 1. Then, one has

$$
V=\frac{1}{r}+h r
$$

for a function $h \in C^{2, \alpha}(M) \cap C^{0}(\bar{M})$ such that

$$
h_{\mid \partial M}=\frac{R_{\gamma}}{4(n-1)(n-2)}, \quad|\nabla h|=O\left(r^{\frac{\alpha}{2}}\right),
$$

where $\alpha>0$ and $R_{\gamma}$ is the scalar curvature of the metric $\gamma$ on $\partial M$. 
Proof. This result relies on some of the assertions in Proposition 4.1, Lemma 5.1 (see Lemma 1 of this paper) and Lemma 5.2 in [35]. These assertions only require $(M, g)$ to be ALH, a condition satisfied by $(M, g)$ since it is the spatial slice of an ALAdS static vacuum. The proof of the second of these lemmae in [35] definitely uses the hypothesis that $(M, g)$ is Einstein. So in fact, we show now that the result holds under weaker hypotheses.

A careful look at the proof of Lemma 5.2 in [35], yields to the observation that the Einstein condition on the metric $g$ is used to obtain the equalities labelled (5.3) and (5.7) in [35], namely

$$
\left\{\begin{array}{l}
(5.3) \quad \widehat{\Delta} r=-\frac{1}{2(n-1)} r R_{\widehat{g}} \quad \text { on a neighbourhood of } \partial M, \\
(5.7) \quad R_{\gamma}=\frac{n-2}{n-1} R_{\widehat{g} \mid \partial M},
\end{array}\right.
$$

where $\widehat{g}=r^{2} g$ is the conformal metric extended to $\bar{M}, \gamma=\widehat{g}_{\mid \partial M}$ its restriction to the conformal infinity and $\widehat{\Delta}$ its scalar Laplacian.

To get (5.3), consider the relation between the Ricci tensors Ric $_{\widehat{g}}$ and Ric $_{g}$ of the conformal metrics $\widehat{g}$ and $g$ on the open manifold $M$ (see again $[12$, p. 59] or [5, Appendix]):

$$
\operatorname{Ric}_{g}=\operatorname{Ric}_{\widehat{g}}+(n-2) \frac{\widehat{\nabla}^{2} r}{r}+\frac{\widehat{\Delta} r}{r} \widehat{g}-(n-1) \frac{|\widehat{\nabla} r|^{2}}{r^{2}} \widehat{g},
$$

where $\widehat{\nabla}$ and $\widehat{\nabla}^{2}$ are respectively the gradient and the Hessian operator of $\widehat{g}$. Multiplying by $r$ and putting $|\widehat{\nabla} r|^{2}=1$, we obtain

$$
r\left(\operatorname{Ric}_{g}+(n-1) g\right)=r \operatorname{Ric}_{\widehat{g}}+(n-2) \widehat{\nabla}^{2} r+(\widehat{\Delta} r) \widehat{g}
$$

Taking traces with respect to $\widehat{g}$, knowing that $R_{g}=-n(n-1)$, it follows that on a collar neighbourhood of $\partial M$ one has

$$
r R_{\widehat{g}}+2(n-1) \widehat{\Delta} r=\frac{1}{r}\left(R_{g}+n(n-1)\right)=0,
$$

giving (5.3) in [35].

As for the second, putting first $r=0$ in (10), then dividing this same expression by $r$ and taking limits as $r \rightarrow 0$, we get

$$
(\widehat{\Delta} r)_{\mid \partial M}=0, \quad(\widehat{\nabla} r \cdot \widehat{\Delta} r)_{\mid \partial M}=-\frac{1}{2(n-1)} R_{\widehat{g} \mid \partial M} .
$$

On the other hand, in the collar neighbourhood of $\partial M$, taking derivatives of $|\widehat{\nabla} r|^{2}=1$ with respect to a vector field $X$ tangent to $\bar{M}$, yield 
$\left(\widehat{\nabla}^{2} r\right)(\widehat{\nabla} r, X)=0$. In particular,

$$
\left(\widehat{\nabla}^{2} r\right)(\widehat{\nabla} r, \widehat{\nabla} r)=0
$$

Again, taking derivatives of $(\widehat{\nabla} d r)(\widehat{\nabla} r, X)=0$ with respect to the same direction $X$, using the Ricci equation and then taking traces, imply

$$
|\widehat{\nabla} d r|^{2}+\widehat{\nabla} r \cdot \widehat{\Delta} r+\operatorname{Ric}_{\widehat{g}}(\widehat{\nabla} r, \widehat{\nabla} r)=0
$$

Now, we apply the tensorial equality (9) to the vector field $\widehat{\nabla} r$ and use (12), to get

$$
\frac{1}{r}\left(\operatorname{Ric}_{g}+(n-1) g\right)\left(\frac{\nabla r}{r}, \frac{\nabla r}{r}\right)=r \operatorname{Ric}_{\widehat{g}}(\widehat{\nabla} r, \widehat{\nabla} r)+\widehat{\Delta} r
$$

Dividing also (14) by $r$, taking limits as $r \rightarrow 0$, keeping in mind the first equality in (11) and using Lemma 4, we obtain

$$
(\widehat{\nabla} r \cdot \widehat{\Delta} r)_{\mid \partial M}=-\operatorname{Ric}_{\widehat{g}}(\widehat{\nabla} r, \widehat{\nabla} r)_{\mid \partial M}
$$

This together with (13) and the second equality in (11) give

$$
\widehat{\nabla}^{2} r_{\mid \partial M}=0, \quad \operatorname{Ric}_{\widehat{g}}(\widehat{\nabla} r, \widehat{\nabla} r)_{\mid \partial M}=\frac{1}{2(n-1)} R_{\widehat{g}}{ }_{\mid \partial M}
$$

Now, take into account that the Hessian $\widehat{\nabla}^{2} r$, restricted to directions orthogonal to the gradient $\widehat{\nabla} r$, is the opposite of the second fundamental form $\widehat{\sigma}$ of the level hypersurfaces $r=r_{0}$ with respect to the choice of inner unit normal $\widehat{N}=\widehat{\nabla} r$ and to the metric $\widehat{g}$ on $\bar{M}$. So, using (12), we see that $\widehat{H}=-\frac{1}{n-1} \widehat{\Delta} r$ is the mean curvature function of these level hypersurfaces. Using such submanifold theory language, we may rewrite the last relations as

$$
\widehat{\sigma}_{\mid \partial M}=0, \quad \widehat{H}_{\mid \partial M}=0, \quad \operatorname{Ric}_{\widehat{g}}(\widehat{N}, \widehat{N})_{\mid \partial M}=\frac{R_{\widehat{g}} \mid \partial M}{2(n-1)} .
$$

Knowing that the Gauß equation relating the scalar curvature $R_{\gamma}$ of the metric $\gamma$ induced on the boundary $\partial M$ and the restriction $R_{\widehat{g} \mid \partial M}$ is given by

$$
R_{\gamma}=R_{\widehat{g} \mid \partial M}-2 \operatorname{Ric}_{\widehat{g}}(\widehat{N}, \widehat{N})_{\mid \partial M}+(n-1)^{2} \widehat{H}_{\mid \partial M}^{2}-|\widehat{\sigma}|_{\mid \partial M}^{2},
$$

the relations in (15) imply equality (5.7) in [35]. 
As mentioned before, the idea of using the eigenfunctions of the Laplacian, with controlled behaviour at infinity, as defining functions on a given ALH Einstein space $(M, g)$ is due to J. Qing (see [37]), although he conformally modifies the original complete manifold through these eigenfunctions without actually compactifying it. Instead, he gets a partial compactification, that is, a conformal complete Riemannian manifold $(\bar{M}, \bar{g})$ whose boundary $\partial M$ is diffeomorphic to the Euclidean space $\mathbb{R}^{n}$ and such that the Riemannian manifold constructed by doubling $(\bar{M}, \bar{g})$ along this boundary is an asymptotically Euclidean manifold without boundary and with nonnegative integrable scalar curvature. Then a suitable use of the positive mass theorem allows him to go on with his reasoning.

Since our ALH manifold $(M, g)$ is the spatial slice of a static vacuum $(M, g, V)$, we know from (2) that $V$ itself is such an eigenfunction of the Laplacian. Moreover, Proposition 5 gives a reasonable control on $V$ near the conformal spatial infinity of $(M, g)$. As in Lemma 3, we will proceed by slightly modifying $V$ in order to totally compactify $(M, g)$.

Theorem 6. Let $(M, g, V)$ be an $(n+1)$-dimensional, $n \geq 3$, ALAdS static vacuum and suppose that the spatial slice $(\partial M, \gamma)$ of its infinity has nonnegative scalar curvature. Then there exists a defining function $\rho^{*}$ for $(M, g)$ such that, if $g^{*}$ is the extension of $\left(\rho^{*}\right)^{2} g$ to $\bar{M}$,

$$
g^{*}{ }_{\mid \partial M}=\gamma, \quad R_{g^{*}} \geq 0, \quad H^{*}=\varepsilon,
$$

where $R_{g^{*}}$ is the scalar curvature of the compact Riemannian manifold $\left(\bar{M}, g^{*}\right), H^{*}$ is the (inner) mean curvature of the conformal infinity $\partial M$ as a hypersurface of $\left(\bar{M}, g^{*}\right)$ and $\varepsilon \geq 0$ is given by

$$
\varepsilon=\inf _{\partial M} \sqrt{\frac{R_{\gamma}}{(n-1)(n-2)}} .
$$

Proof. As in Lemma 3, we define the function $\rho^{*}=(V+\varepsilon)^{-1}$ for this precise choice of $\varepsilon$. In Lemma 3 we also showed that $\rho^{*}$ is indeed a defining function for $(M, g)$ with $g^{*}{ }_{\mid \partial M}=\gamma$ and established that the scalar curvature of the compact Riemannian with boundary $\left(\bar{M}, g^{*}\right)$, satisfies (4).

Now, to show that $R_{g^{*}} \geq 0$, our approach is to study the points where the function $\Phi:=V^{2}-|\nabla V|^{2}-\varepsilon^{2}$, defined on $\bar{M}$, attains its minimum. Taking into account the Bochner formula for the Laplacian of the squared length of 
a gradient, we have

$$
\frac{1}{2} \Delta \Phi=|\nabla V|^{2}+V \Delta V-\left|\nabla^{2} V\right|^{2}-g(\nabla V, \nabla \Delta V)-\operatorname{Ric}_{g}(\nabla V, \nabla V) .
$$

Using (1), we obtain

$$
\frac{1}{2} \Delta \Phi=-\left|\nabla^{2} V-V g\right|^{2}-\frac{\left(\nabla^{2} V\right)(\nabla V, \nabla V)}{V}+|\nabla V|^{2} .
$$

On the other hand, from the definition of $\Phi$, we have

$$
g(\nabla \Phi, \nabla V)=V|\nabla V|^{2}-\left(\nabla^{2} V\right)(\nabla V, \nabla V) .
$$

If the minimum of $\Phi$ is attained at a point $p \in M$, then (17) and (16) would imply that $(\Delta \Phi)(p) \leq 0$ and thus the strong minimum principle implies that $\Phi$ is constant. Consequently, the minimum of $\Phi$ could be also attained at the infinity $\partial M$. Hence, we can assume that $\Phi$ reaches its minimum value at $\partial M$.

To study the asymptotical behaviour of the function $\Phi$, we can use the expression (7) of the eigenfunction $V$ in terms of the geodesic defining function $r$. Then

$$
\nabla V=\left(h-\frac{1}{r^{2}}\right) \nabla r+r \nabla h
$$

Taking squared norms with respect to the metric $g$, we get

$$
|\nabla V|^{2}=\left(h-\frac{1}{r^{2}}\right)^{2}|\nabla r|^{2}+r^{2}|\nabla h|^{2}+2 r\left(h-\frac{1}{r^{2}}\right) g(\nabla r, \nabla h) .
$$

But we know that $|\nabla r|^{2}=r^{2}$ near $\partial M$ due to the geodesic character of the defining function $r$. Putting this into the last equation and using (7) again, we have

$$
\Phi=4 h-r^{2}|\nabla h|^{2}-2 r\left(h-\frac{1}{r^{2}}\right) g(\nabla r, \nabla h),
$$

which is valid in a collar neighbourhood of $\partial M$. From (8), we know that $h$ extends to $C^{0}(\bar{M})$ and that $|\nabla h|=O\left(r^{\frac{\alpha}{2}}\right)$. So, the third term in the right side of the previous equation satisfies

$$
\left|r\left(h-\frac{1}{r^{2}}\right) g(\nabla r, \nabla h)\right| \leq\left|h r^{2}-1\right||\nabla h|=O\left(r^{\frac{\alpha}{2}}\right)
$$

as a consequence of the Schwarz inequality for $g$ and again from the fact that $|\nabla r|=r$ in a collar neighbourhood of $\partial M$. From this inequality and 
the equality above, we conclude, taking limits when $r \rightarrow 0$, that

$$
\Phi_{\mid \partial M}=\left(V^{2}-|\nabla V|^{2}\right)_{\mid \partial M}=4 h_{\mid \partial M}=\frac{R_{\gamma}}{(n-1)(n-2)} \geq \varepsilon^{2},
$$

where we have used again (8) and the choice of $\varepsilon$.

Thus $\Phi_{\mid \partial M} \geq \varepsilon^{2}$ together with equality (4) imply that on $\bar{M}$, one has

$$
R_{g^{*}}=n(n-1)\left(V^{2}-|\nabla V|^{2}-\varepsilon^{2}\right) \geq 0 .
$$

To finish the proof, it remains to compute the mean curvature $H^{*}$ of the conformal infinity $\partial M$ as a hypersurface of the compactified Riemannian manifold $\left(\bar{M}, g^{*}\right)$. Observe that, by definition of $\rho^{*}$ and Proposition 5 ,

$$
g^{*}=\left(\rho^{*}\right)^{2} g=\left(\frac{r}{1+\varepsilon r+h r^{2}}\right)^{2} g=\left(\frac{1}{1+\varepsilon r+h r^{2}}\right)^{2} \widehat{g}
$$

where $\widehat{g}=r^{2} g$ is the extended metric on $\bar{M}$ corresponding to the geodesic defining function $r$. But, we know from (15) that the mean curvature $\widehat{H}$ of the spatial conformal infinity $\partial M$ as a hypersurface of $(\bar{M}, \widehat{g})$ vanishes (in fact, it is a totally geodesic hypersurface). So, in order to compute $H^{*}$, it suffices to use the well-known relation between the two mean curvatures of a hypersurface corresponding to two conformal metrics on the ambient space (see, for instance, $[31,(4.4)])$ :

$$
H^{*}=\frac{1}{R}(\widehat{H}-\widehat{g}(\widehat{\nabla} \log R, \widehat{N}))=-\frac{1}{R^{2}} \widehat{g}(\widehat{\nabla} R, \widehat{N}),
$$

where $R=\left(1+\varepsilon r+h r^{2}\right)^{-1}$ and $\widehat{N}$ is the inner unit normal along $\partial M$ with respect to the metric $\widehat{g}$, which can be also written as $\widehat{\nabla} r$. Since

$$
\widehat{\nabla} R_{\mid \partial M}=-\left.\frac{\varepsilon \widehat{\nabla} r+\widehat{\nabla}\left(r^{2} h\right)}{\left(1+\varepsilon r+h r^{2}\right)^{2}}\right|_{\partial M}=-\varepsilon \widehat{\nabla} r_{\mid \partial M},
$$

we finally obtain

$$
\left(H^{*}\right)_{\mid \partial M}=\varepsilon \widehat{g}(\widehat{\nabla} r, \widehat{\nabla} r)_{\mid \partial M}=\varepsilon .
$$




\section{Spatial slice admitting Killing or parallel spinors on its infinity}

Suppose now that the spatial slice $M$ of an $\operatorname{ALAdS~static~vacuum~}(M, g, V)$ is a spin manifold on which we fix a spin structure. We know that its spatial slice $(M, g)$ is an ALH manifold. Indeed, the metric $\bar{g}=V^{-2} g$ on $M$ extends to a compact manifold with boundary $\bar{M}$ whose interior is $M$ itself. Then, it is straightforward to check that $\bar{M}$ is also a spin manifold and that we may fix a unique spin structure on $\bar{M}$ such that its restriction on the open subset $M$ is precisely the given spin structure of $M$. Since the spatial conformal infinity $\partial M$ is always an orientable hypersurface (recall that the gradient of a geodesic defining function provides a global unit normal field), we have that $\partial M$ is also a spin manifold and that an induced spin structure on the infinity is inherited from the fixed structure on $\bar{M}$. Moreover, for the Riemanian metric $\bar{g}$ on $\bar{M}$ we have an associated spinor bundle $(\mathbb{S} \bar{M}, \bar{\nabla}, \overline{\mathfrak{c}})$, where $\bar{\nabla}$ is the spin Levi-Civita connection and $\overline{\mathfrak{c}}$ is the Clifford multiplication (for generalities on spin structures see any of $[11,16,23,25,34]$ ). It is a well-known fact that the restriction to the hypersurface $\partial M$ of the spinor bundle $\mathbb{S} \bar{M}$ can be identified with one or two copies of the spinor bundle corresponding to the induced spin structure and the induced Riemannian metric $\gamma=\bar{g}_{\mid \partial M}$ according to the parity of the dimension $n$ of $\bar{M}$. More precisely, if $\varphi$ is a section of the restricted bundle $\mathbb{S} \bar{M}_{\mid \partial M}$, we consider the new Clifford multiplication

$$
\mathfrak{c}^{\partial M}(X) \varphi=\overline{\mathfrak{c}}(X) \overline{\mathfrak{c}}(N) \varphi
$$

and the new connection

$$
\nabla_{X}^{\partial M} \varphi=\bar{\nabla}_{X} \varphi-\frac{1}{2} \overline{\mathfrak{c}}(\bar{A} X) \overline{\mathfrak{c}}(\bar{N}) \varphi=\bar{\nabla}_{X} \varphi-\frac{1}{2} \mathfrak{c}^{\partial M}(\bar{A} X) \varphi, \quad \forall X \in \Gamma(T \partial M),
$$

where $\bar{N}$ is the (inner) unit normal field along $\partial M$ and $\bar{A}$ is its corresponding shape operator. Then, we have an isomorphism

$$
\left(\mathbb{S} \bar{M}_{\mid \partial M}, \nabla^{\partial M}, \mathfrak{c}^{\partial M}\right) \cong\left\{\begin{array}{l}
(\mathbb{S} \partial M, \nabla, \mathfrak{c}), \text { if } n \text { is odd } \\
(\mathbb{S} \partial M, \nabla, \mathfrak{c}) \oplus(\mathbb{S} \partial M, \nabla,-\mathfrak{c}), \text { if } n \text { is even }
\end{array}\right.
$$

where $(\mathbb{S} \partial M, \nabla, \mathfrak{c})$ is the spinor bundle corresponding to the spin structure and to the Riemannian metric induced on $\partial M$ (for this relationship between the spinor bundles on a hypersurface and on its ambient space, see, for instance, $[9,23,28,30,31])$. Due to this identification we can say that each 
spinor field on $\bar{M}$ determines, by restriction, a spinor field on the boundary $\partial M$ and we can talk about possible extensions to $\bar{M}$ of the spinor fields defined on $\partial M$.

Let $\varphi \in \Gamma(\mathbb{S} \partial M)$ be a spinor field on the Riemannian slice of the conformal null infinity of an ALAdS spin static vacuum $(M, g, V)$. When $\varphi$ satisfies the first order equation

$$
\nabla_{X} \varphi+\frac{\lambda}{n-1} \mathfrak{c}(X) \varphi=0, \quad \lambda \in \mathbb{C}, \quad \forall X \in \Gamma(T \partial M),
$$

we will say that $\varphi$ is a Killing spinor if $\lambda \in \mathbb{C}^{*}$. Of course, if the same equation is satisfied with $\lambda=0$, we say that $\varphi$ is a parallel spinor (we refer to $[11,16,17,25]$ for definitions and main properties). It can be shown that $\lambda$ has to be a real or a purely imaginary number. So, we will talk about real or imaginary Killing spinors according to $\lambda \in \mathbb{R}^{*}$ or $\lambda \in i \mathbb{R}^{*}$. Of course, these definitions are usually made for general spin Riemannian manifolds, not necessarily boundaries of compactifications. It is immediate that a Killing or parallel spinor must be an eigenspinor for the well-known Dirac operator $D$ locally defined by

$$
D \varphi=\sum_{i=1}^{n-1} \mathfrak{c}\left(e_{i}\right) \nabla_{e_{i}} \varphi
$$

where $\left\{e_{1}, \ldots, e_{n-1}\right\}$ is a local orthonormal frame on $\partial M$. In fact, (19) immediately implies $D \varphi=\lambda \varphi$.

The existence of parallel or Killing spinors imposes strong restrictions on the geometry of the manifold and on its holonomy. Such manifolds have to be Einstein with scalar curvature $R=4(n-1)(n-2) \lambda^{2}$. Indeed, M. Wang, H. Baum and C. Bär ([7, 8, 10, 38, 39]) classified some types of spin Riemannian manifolds admitting non-trivial parallel, imaginary Killing and real Killing spinors, respectively. When the considered spin Riemannian manifold is compact, since the eigenvalues of its Dirac operator have to be real, Killing spinors must be real as well and, moreover, as it was shown by $\mathrm{T}$. Friedrich [11, Corollary 1, Theorem 9], they are eigenspinors corresponding to the eigenvalues with the least absolute value $\pm \sqrt{\frac{(n-1) R}{4(n-2)}}$. This quick review about spin structures and Killing spinors allows us to set up our first rigidity result.

Theorem 7. Let $(M, g, V)$ be an $(n+1)$-dimensional ALAdS static vacuum. Suppose that $M$ is a spin manifold. 
1) If the boundary at infinity $(\partial M, \gamma)$ admits a non-trivial Killing, then $(M, g, V)$ is the AdS spacetime.

2) The boundary at infinity $(\partial M, \gamma)$ cannot admit a non-trivial parallel spinor.

We emphasize that the spin structure considered on $\partial M$ is just the induced one from that of $M$.

Proof. Take a non-trivial Killing or parallel spinor $\varphi \in \Gamma(\mathbb{S} \partial M)$ on the boundary at infinity $(\partial M, \gamma)$ of the vacuum $(M, g, V)$. Since $\partial M$ is compact, we know that $\lambda$ in (19) is a real number and that the metric $\gamma$ on $\partial M$ has constant scalar curvature $R_{\gamma}=(n-1)(n-2) \lambda^{2}$. So, we have on $\partial M$ either positive constant scalar curvature and a non-trivial real Killing spinor $\varphi_{1}$, or identically zero scalar curvature and a non-trivial parallel spinor $\varphi_{0}$. In particular, we can apply Theorem 6 to this situation by choosing $\varepsilon=|\lambda|$. So we have a metric $g^{*}$ on the spin compactification $\bar{M}$ such that $R_{g^{*}} \geq 0$, $H^{*}=\varepsilon$ and $g^{*}{ }_{\mid \partial M}=\gamma$. Under these conditions, we may use a lower estimate for the spectrum of the Dirac operator $D$ of $(\partial M, \gamma)$ obtained by X. Zhang and the authors in [30] (see [25, Theorem 3.7.1]). This result asserts that, if $\lambda_{1}(D)$ stands for the eigenvalue of $D$ with the lowest absolute value, then

$$
\left|\lambda_{1}(D)\right| \geq \frac{n-1}{2} \varepsilon
$$

and, if the equality holds, then the eigenspace associated with $\lambda_{1}(D)$ is built from parallel spinor fields on $\left(\bar{M}, g^{*}\right)$. But we know that there exists on $\partial M$ a non-trivial Killing or parallel spinor $\varphi_{i(\varepsilon)}$, with $i(\varepsilon)=1$ or $i(\varepsilon)=0$, depending on whether $\varepsilon>0$ or $\varepsilon=0$ and hence $D \varphi_{i(\varepsilon)}= \pm \frac{n-1}{2} \varepsilon \varphi_{i(\varepsilon)}$. Then the equality $\left|\lambda_{1}(D)\right|=\frac{n-1}{2} \varepsilon$ holds and so $\varphi_{i(\varepsilon)}$ comes from a parallel spinor field $\Psi \in \mathbb{S} \bar{M}$. Note that $\Psi$ has to be a non-trivial parallel spinor since its restriction to $\partial M$ is non-trivial.

It was shown by Hitchin in [32] (see also [11, Chapter 6]) that the existence of a non-trivial parallel spinor forces the Ricci tensor to vanish identically. Then $\mathrm{Ric}_{g^{*}}=0$ on $\bar{M}$ and so $R_{g^{*}}=0$ as well. From (4), (16), (17) and (18), and the proof of Theorem 6 , we conclude

$$
\nabla^{2} V=V g, \quad V^{2}-|\nabla V|^{2}-\varepsilon^{2}=0
$$

Hence the complete manifold $(M, g)$ admits a non-trivial (in fact, positive) solution $V$ to the Obata type equation $\nabla^{2} V=V g$. If the function $V$ has a critical point, Theorem $\mathrm{C}$ in [33] implies that $(M, g)$ is isometric to the 
hyperbolic space $\left(\mathbb{H}^{n}, g_{\mathbb{H}}\right)$ and $V$ is a positive height function. Then $\left(\mathcal{V}, g_{\mathcal{V}}\right)$ is isometric to the AdS spacetime. Assume on the contrary that $V$ has no critical points on $M$. We can normalize the gradient $\nabla V$ to obtain, as a consequence of (20), a global unit vector field $X=\frac{\nabla V}{|\nabla V|}$ on $M$ satisfying $\nabla_{X} X=0$. Hence the integral curves of $X$ are geodesics and are defined on the whole real line. Take a positive real number $a$ in the image of $V$. Then $P=V^{-1}(\{a\})$ is a closed hypersurface in $M$ and so compact because $V$ tends to $+\infty$ when one approaches $\partial M$ (see (7)). Let $\mathcal{F}: M \times \mathbb{R} \rightarrow M$ be the flow of $X$. From the considerations above it follows that the restriction $\mathcal{F}: P \times \mathbb{R} \rightarrow M$ is a diffeomorphism with

$$
\mathcal{F}(p, s)=\gamma_{p}(s), \quad \forall p \in P, \forall s \in \mathbb{R}
$$

where $\gamma_{p}: \mathbb{R} \rightarrow M$ is the integral (geodesic) curve of $X$ with initial condition $\gamma_{p}(0)=p$. In particular, $P$ must be connected. Moreover, if $Y \in \Gamma(T P)$ is a vector field tangent to $P$, we have $Y \cdot V=g(\nabla V, Y)=0$, because the gradient $\nabla V$ is orthogonal to the level hypersurfaces. This means that $V(\mathcal{F}(p, s))$ depends only on $s$. On the other hand, Equation (20) implies that

$$
\left(V \circ \gamma_{p}\right)^{\prime \prime}(s)=V\left(\gamma_{p}(s)\right), \quad V\left(\gamma_{p}(s)\right)^{2}-\left(\left(V \circ \gamma_{p}\right)^{\prime}(s)\right)^{2}-\varepsilon^{2}=0,
$$

for each $p \in P$ and $s \in \mathbb{R}$. It follows that

$$
V\left(\gamma_{p}(s)\right)=a \cosh s+b \sinh s, \quad b \in \mathbb{R} \quad \text { with } \quad a^{2}=b^{2}+\varepsilon^{2}
$$

Since we are assuming that $V$ has no critical points on $M$ and $V\left(\gamma_{p}(s)\right)$ only depends on $s$, we deduce that

$$
\left(V \circ \gamma_{p}\right)^{\prime}(s)=a \sinh s+b \cosh s \neq 0, \quad \forall s \in \mathbb{R},
$$

and this is equivalent to the inequality $|b| \geq a$. It turns out that $|b|=a>0$ and $\varepsilon=0$ and so, reversing the parameter $s$ if necessary, we obtain

$$
V\left(\gamma_{p}(s)\right)=a e^{s}, \quad \forall p \in P, \forall s \in \mathbb{R}
$$

But, from (7), we know that $V\left(\gamma_{p}(s)\right) \rightarrow+\infty$ when $\gamma_{p}(s)$ approaches $\partial M$. Hence

$$
\exists \lim _{s \rightarrow-\infty} \gamma_{p}(s)=\gamma_{p}(-\infty) \in M \quad \text { and } \quad V\left(\gamma_{p}(-\infty)\right)=0,
$$

which is a contradiction, since $V$ is positive. 
Remark 1. If the proof of Theorem 7 is closely analyzed, one can see that we actually show that, for each Killing or parallel spinor on the spatial infinity $(\partial M, \gamma)$, there exists an imaginary Killing spinor on the bulk spatial manifold $(M, g)$. In fact, we have proved that there exists a parallel spinor $\Psi \in \Gamma(\mathbb{S} \bar{M})$ on the conformal compactification $\left(\bar{M}, g^{*}\right)$. Now, the same construction which allows to pass from parallel Euclidean spinors to imaginary hyperbolic Killing spinors by using the conformal factor between the Euclidean and the hyperbolic metrics on the disc $B^{n+1}$ (see $[11,25]$ ) can be used in order to build from $\Psi$ an imaginary Killing spinor on $(M, g)$. This means that we can extend each supersymmetric infinitesimal isometry of $(\partial M, \gamma)$ to a supersymmetric infinitesimal isometry of the spatial slice $(M, g)$ of the ALAdS static vacuum. This is an analogue to the result of extending conformal transformations on the conformal infinity of an ALH Einstein manifold to isometries on the bulk manifold proved by M. Anderson (see [3, Theorem 3.3] and [5, Theorem 2.5]). In fact, our result gives rise to the following questions: Is it possible to extend each Killing vector field on $(\partial M, \gamma)$ to a Killing vector field on $(M, g)$ for the spatial slice of any ALAdS static vacuum? What about isometries? In fact, the infinitesimal question was already treated by X. Wang in [40, Section 4, Theorem 4.1], by proving that the extension is possible for ALH Einstein manifolds with non-positive sectional curvature.

Notice that in this supersymmetric setup for ALAdS static vacua, the conclusion from the existence of only one Killing or parallel spinor is stronger than the existence of a conformal transformation in M. Anderson's result: the presence of only one such spinor on the boundary at infinity forces the bulk manifold to be maximally symmetric. We will point out that this occurs because we prevent our vacua $(M, g, V)$ to have singularities. By the way, from the existence of the aforementioned imaginary Killing spinor on $(M, g)$ and Baum's classification given in [10] we could finish the proof in an alternative way, but other considerations led us to adopt our approach.

Due to its simply-connectedness, the sphere $\mathbb{S}^{n-1}$ has a unique spin structure. Moreover, the spinor bundle corresponding to this structure and to the round Riemannian metric has a $2^{\left[\frac{n-1}{2}\right]}$-dimensional space of real Killing spinors for $\lambda=\frac{n-1}{2}$ and another one with the same dimension for $\lambda=-\frac{n-1}{2}$ (see [11, p. 37], [25, Examples A.1.3.2]). In this way we see that Theorem 7 implies the rigidity result by X. Wang and by P. Chruściel and M. Herzlich.

Corollary 8. Let $(M, g, V)$ be an $(n+1)$-dimensional, $n \geq 3$, AAdS static vacuum. Suppose that $M$ is a spin manifold and that the infinity $(\partial M, \gamma)$ 
of its spatial slice $(M, g)$ is a unit round hypersphere. Then $(M, g, V)$ is the AdS spacetime.

Besides this uniqueness of the hyperbolic space when the prescribed conformal infinity of the spatial slices is a round sphere, Theorem 7 provides a non-existence result when this conformal infinity is a non-spherical compact spin manifold carrying non-trivial real Killing spinors. In the simplyconnected case, C. Bär determined in [7] all these spin manifolds (see also [1] and [8, Theorem 3] for non-simply-connected space forms). Using this classification, we obtain the following:

Corollary 9. There is no $(n+1)$-dimensional, $n \geq 3$, ALAdS static vacua $(M, g, V)$ whose spatial slices have infinities $(\partial M, \gamma)$ isometric to non-spherical compact spin manifolds admitting a non-trivial real Killing spinor. In the simply-connected case, they are Einstein-Sasaki manifolds, 3-Sasaki manifolds, nearly-Kähler non-Kähler 6-manifolds and 7-manifolds carrying nice 3-forms. In the non-simply-connected case, they include, for example, all the round quotients $\mathbb{S}^{3} / \Gamma$, where $\Gamma \subset \mathbb{S}^{3}$ is any of its finite subgroups, and real projective spaces $\mathbb{R} P^{n}$ with dimensions $n=8 k+3$ or $n=8 k+7$, $k \geq 0$.

Theorem 7 also provides a non-existence result when $(\partial M, \gamma)$ is isometric to a compact spin manifold admitting non-trivial parallel spinors. Taking into account that the product of spin manifolds with non-trivial parallel spinors is also another spin manifold of this same type, and that M. Wang determined in $[38,39]$ all irreducible spin manifolds carrying parallel spinors (see also [25, Theorem A.4.2]), we obtain the following:

Corollary 10. There is no $(n+1)$-dimensional, $n \geq 3$, ALAdS static vacua $(M, g, V)$ whose conformal null infinities $\mathcal{J}$ have spatial slices $\left(\partial M, V^{-2} g_{\mid \partial M}\right)$ isometric to compact spin manifolds admitting a non-trivial parallel spinor. In the simply-connected case, they are just Calabi-Yau manifolds, hyperKähler manifolds, $G_{2}$ 7-manifolds, Spin(7) 8-manifolds and all their Riemannian products. In the non-simply-connected case, they include, for example, all the flat tori $\mathbb{T}^{n}$ with the trivial spin structure and all the Riemannian products of trivial flat tori $\mathbb{T}^{k}, 1 \leq k \leq n-2$, with the examples of simplyconnected manifolds above.

Remark 2. It is important to note that the role of spin structures is essential. In fact, it is well-known that the so-called family of AdS toroidal black hole metrics (see [14] or [3, Example 2.2, Remark 3.4 ii)]), constructed on the 
solid $n$-dimensional torus $B^{2} \times \mathbb{T}^{n-2}$ are the unique ALH Einstein manifolds whose conformal infinity is the flat torus $\mathbb{T}^{n-1}$. They are given by

$$
g=\frac{1}{U(r)} d r^{2}+U(r) d \theta^{2}+r^{2} g_{\mathbb{T}^{n-2}},
$$

where $g_{\mathbb{T}^{n-2}}$ is the standard flat metric on the torus and

$$
U(r)=r^{2}\left(1-\frac{r_{0}^{n}}{r^{n}}\right)
$$

with $r_{0}>0$ and $\theta$ a $\left(4 \pi / n r_{0}\right)$-periodic coordinate. One can check that, taking $V=V(r)=r^{2}$, we have that $\left(B^{2} \times \mathbb{T}^{n-2}, g, V\right)$ is a static vacuum. Indeed it determines the so-called AdS soliton (see [42] and [24] for a uniqueness result). It is clear that $M=B^{2} \times \mathbb{T}^{n-2}$ is a spin manifold, but the spin structure inherited on its boundary at infinity $\partial M=\mathbb{T}^{n-1}$ is not the trivial one, since $M$ is constructed by gluing on a 2-disc onto a simple closed geodesic of the flat $\mathbb{T}^{n-1}$. Then, even though $(\partial M, \gamma)$ is a flat torus, the spin structure inherited from $(M, g)$ admits no parallel spinor fields and Corollary 10 does not apply in this situation.

\section{Supersymmetries on the boundary and spin conformal compactifications with singularities}

If we compare Theorem 3.3 in [3] (see also Theorem 2.5 in [5]) with the main Theorem 7 of this paper, we see that, in the spirit of the AdS/CFT correspondence, all the conformal transformations on the boundary of an ALH Einstein manifold come from Riemannian isometries of its conformal compactifications and that, however, in the case of ALAdS static vacua, the supersymmetric infinitesimal isometries of the null infinity give nonexistence results, except for the spherical case.

Hence, the proof of Theorem 7 can be read as a supersymmetric version of Anderson's result under different conditions on the Ricci curvature. Indeed, its proof shows that each Killing or parallel spinor on the spatial slice of the null infinity comes from an imaginary Killing spinor on the bulk manifold (see Remark 1). But a careful reading of this proof makes also manifest that, if we exclude conical or cusp singularities, the only vacua supporting these imaginary Killing spinors are AdS spacetimes. This is why we finish this paper with some examples showing that there are ALAdS static vacua with null infinities carrying Killing or parallel spinors provided that singularities are allowed. 
We could say that supersymmetries on a non-spherical null infinity yield static vacua with hyperbolic conical or cusps singularities. Mathematically, these examples take the form of warped products. From a physical point of view such kind of manifolds also appear regularly $([6,14])$. On the other hand, a strong necessity of considering ALAdS metrics with singularities arises as well when one wants to understand the manifold structure of the space of these metrics, with a given topology (see, for example, $[3,(3.6)]$ ).

Example 1. Consider the Poincaré hyperbolic ball $\left(B^{n}, \frac{4|d x|^{2}}{\left(1-|x|^{2}\right)^{2}}\right)$ as a model of the hyperbolic space. Using polar coordinates $x=r p$, with $r \in] 0,1$ ] and $p \in \mathbb{S}^{n-1}$, it follows that the hyperbolic metric of constant sectional curvature -1 takes the form

$$
g=\left(\frac{2}{1-r^{2}}\right)^{2}\left(d r^{2}+r^{2} \gamma_{\mathbb{S}^{n-1}}\right)
$$

where $\gamma_{\mathbb{S}^{n-1}}$ stands for the unit round metric on the sphere. If we consider the change of variables given by $s=\ln \frac{1+r}{1-r} \in \mathbb{R}^{+}$, we obtain

$$
g=d s^{2}+\left(\sinh ^{2} s\right) \gamma_{\mathbb{S}^{n-1}}
$$

These two expressions for the Poincaré metric are valid only on the punctured ball $\left.B^{n}-\{0\} \cong\right] 0,1\left[\times \mathbb{S}^{n-1} \cong \mathbb{R}^{+} \times \mathbb{S}^{n-1}\right.$, although they are smoothly extendable to the origin. This latter is an example of the so-called warped Riemannian products (see, for instance, $[12,33,36]$ ).

In general, if $I \subset \mathbb{R}$ is an open interval, $(P, \gamma)$ a Riemannian $(n-1)$ manifold and $f \in C^{\infty}(I)$ is a positive function, we will say that the $n$ dimensional Riemannian manifold $\left(I \times P, g=d s^{2}+f(s)^{2} \gamma\right)$ is the product of $I$ and $P$ warped by means of the function $f$. For the sake of simplicity, if we only consider Einstein manifolds, using the form of the Ricci tensor on a warped product (see [33, Lemma 4]) and recalling (2), we have restricted ourselves to warping functions $f$ satisfying the linear differential equation $f^{\prime \prime}-f=0$. With this choice, we ensure that $\operatorname{Ric}_{g}\left(\frac{\partial}{\partial s}, \frac{\partial}{\partial s}\right)=-(n-1)$ at each point of $I \times P$. Taking into account the values of $\mathrm{Ric}_{g}$ on the directions orthogonal to the vector field $\frac{\partial}{\partial s}$, that is, directions tangent to $P$, we conclude that there are essentially three types of warped products which eventually may produce Einstein manifolds with scalar curvature $-n(n-1)$. The first one is the hyperbolic cone on a given compact Riemannian manifold $(P, \gamma)$, given by

$$
\left(\mathbb{R}^{+} \times P, g=d s^{2}+\left(\sinh ^{2} s\right) \gamma\right)
$$


It is immediate to see again from [33, Lemma 4], for instance, that we have for the directions tangent to this cone and perpendicular to the radial direction

$$
\operatorname{Ric}_{g}+(n-1) g=\operatorname{Ric}_{\gamma}-(n-2) \gamma
$$

So, we will assume that $(P, \gamma)$ is an Einstein manifold with scalar curvature $(n-1)(n-2)$. We can also see in [33] that the smooth function defined on $\mathbb{R}^{+} \times P$ by $V(s, p)=\cosh s$, for each $s \in \mathbb{R}^{+}$and $p \in P$, is a solution to the Obata type equation $\nabla^{2} V=V g$. Thus the triple $(M, g, V)=\left(\mathbb{R}^{+} \times P, d s^{2}+\right.$ $\left.\left(\sinh ^{2} r\right) \gamma, \cosh r\right)$ determines a static vacuum. Defining a new variable $t \in$ ]0, $\left.\frac{\pi}{2}\right]$ by the equality $t=-\frac{\pi}{2}+2 \arctan e^{s}$, we obtain

$$
\bar{g}=\frac{1}{\cosh ^{2} s} g=d t^{2}+\left(\sin t^{2}\right) \gamma
$$

The spherical conical metric $d t^{2}+\left(\sin t^{2}\right) \gamma$ obviously extends to $\left[0, \frac{\pi}{2}\right] \times P$, a compact manifold with boundary $\left\{\frac{\pi}{2}\right\} \times P \cong P$ and a conical singularity at $t=0$. This singularity is removable if and only if $(P, \gamma)$ is the round unit $(n-1)$-sphere and, in this case, the corresponding hyperbolic cone is nothing but the $n$-dimensional hyperbolic space (see $[12$, p. 269 , Lemma 9.114]). When $P$ is chosen to be one of the non-spherical compact spin $(n-1)$-dimensional manifolds listed in Corollary 9, we get an example of non-AdS ALAdS static vacuum with spatial infinity supporting non-trivial Killing spinors with a conical singularity.

Example 2. The second type of warped product relevant to our purposes is the so-called hyperbolic cusp on a compact Riemannian manifold $(P, \gamma)$, given by

$$
\left(\mathbb{R} \times P, g=d s^{2}+e^{2 s} \gamma\right) .
$$

For these cusps, the Ricci curvature is also - $(n-1)$ along the radial direction $\frac{\partial}{\partial s}$ and, for orthogonal directions tangent to $P$, we have

$$
\operatorname{Ric}_{g}+(n-1) g=\operatorname{Ric}_{\gamma}
$$

Then, we will assume that in this case $(P, \gamma)$ is Ricci-flat. As in Example 1, we can see in [33] that $V(s, p)=e^{s}$ is a solution to $\nabla^{2} V=V g$. Defining a new variable $t \in \mathbb{R}^{+}$as $t=e^{s}$, we obtain

$$
\bar{g}=\frac{1}{e^{2 s}} g=d t^{2}+\gamma
$$


The cylindrical metric $d t^{2}+\gamma$ clearly extends to $[0,+\infty[\times P$, which is a noncompact manifold with boundary $\{0\} \times P \cong P$ and one cylindrical end. So, the corresponding static vacuum $(M, g, V)=\left(\mathbb{R}^{+} \times P, d s^{2}+e^{2 s} \gamma, e^{s}\right)$ has a null infinity whose Riemannian slice is $(P, \gamma)$ for $s=+\infty$, but it is not compactifiable at $s=-\infty$ because of the presence of a hyperbolic cusp. Of course, the cusp singularity is always unremovable, although it has finite volume. When $P$ is chosen to be one of the non-spherical compact spin $(n-1)$-dimensional manifolds listed in Corollary 10, we get an example of non-AdS ALAdS static vacuum with spatial infinity supporting non-trivial parallel spinors having a cusp singularity.

\section{References}

[1] B. Ammann, A variational problem in conformal spin geometry, Habilitationsschrift, Universtität Hamburg, 2003.

[2] L. Andersson, M. Dahl, Scalar curvature rigidity for asymptotically locally hyperbolic manifolds, Ann. Glob. Anal. Geom., 16 (1998), 1-27.

[3] M. Anderson, Geometric aspects of the AdS/CFT correspondence, in O. Biquard (ed.), AdS/CFT Correspondence: Einstein Metrics and Their Conformal Boundaries, Euro. Math. Soc., Zurich, (2005), 1-31.

[4] M. Anderson, Topics in conformally compact Einstein metrics, Perspectives in Riemannian Geometry, CRM Proc. Lecture Notes, 40 (2006), 1-26.

[5] M. Anderson, Einstein metrics with prescribed conformal infinity on 4-manifolds, Geom. Funct. Anal., 18 (2008), 2009-2045.

[6] A. Award, A. Chamblin, A bestiary of higher dimensional Taub-NUTAdS spacetimes, Class. Quant. Grav., 19 (2002), 2051-2061.

[7] C. Bär, Real Killing spinors and holonomy, Commun. Math. Phys., 154 (1993), 509-521.

[8] C. Bär, The Dirac operator on space forms of positive curvature, J. Math. Soc. Japan, 48 (1996), 69-83.

[9] C. Bär, Extrinsic bounds of the Dirac operator, Ann. Glob. Anal. Geom., 16 (1998), 573-596.

[10] H. Baum, Complete Riemannian manifolds with imaginary Killing spinors, Ann. Glob. Anal. Geom., 7 (1989), 205-226. 409-422. 
[11] H. Baum, T. Friedrich, R. Grünewald, I. Kath, Twistor and Killing Spinors on Riemannian Manifolds, Teubner-Texte zur Mathematik, Band 124, Teubner-Verlag, Sttugart/Leipzig, 1991.

[12] A. Besse, Einstein manifolds, Springer, New York,(1987).

[13] O. Biquard (ed.), AdS/CFT Correspondence: Einstein Metrics and Their Conformal Boundaries, IRMA Lectures in Mathematics and Theoretical Physics, Euro. Math. Soc., Zürich, 2005.

[14] J. de Boer, L. Maoz, A. Naqvi, Some aspects of the AdS/CFT correspondence, in O. Biquard (ed.), AdS/CFT Correspondence: Einstein Metrics and Their Conformal Boundaries, Euro. Math. Soc., Zurich, (2005), 33-58.

[15] W. Boucher, G. W. Gibbons, G. T. Horowitz, Uniqueness theorem for anti-de Sitter spacetime, Phys. Rev. D, 30 (1984), 2447-2451.

[16] J. P. Bourguignon, O. Hijazi, J. L. Milhorat, A. Moroianu, S. Moroianu, A spinorial approach to Riemannian and conformal geometry, to appear, Monographs in Math., European Mathematical Society, 2014.

[17] M. Cahen, S. Gutt, L. Lemaire, P. Spindel, Killing spinors, Bull. Soc. Math. Belg., 38 (1986), 75-102.

[18] M. Cai, G. J. Galloway, Boundaries of zero scalar curvature in the AdS/CFT correspondence, Adv. Theor. Math. Phys., 3 (1999), 17691783.

[19] P. T. Chruściel, E. Delay, J. M. Lee, D. N. Skinner, Boundary regularity of conformally compact Einstein metrics, J. Diff. Geom., 69 (2005), 111-136.

[20] P. T. Chruściel, M. Herzlich, The mass of asymptotically hyperbolic Riemannian manifolds, Pacific J. Math., 212 (2003), 231-264.

[21] P. T. Chruściel, W. Simon, Towards the classification of static vacuum spacetimes with negative cosmological constant, J. Math. Phys., 42 (2001), 1779-1817.

[22] C. Fefferman, C. R. Graham, Conformal invariants, in "Élie Cartan et les mathématiques d'aujourd'hui", Astérisque, (1985), 95-116.

[23] T. Friedrich, Dirac Operators in Riemannian Geometry, AMS Graduate Studies in Math., vol. 25, 2000. 
[24] G. J. Galloway, S. Surya, E. Woolgar, On the geometry and mass of static, asymptotically AdS spacetimes, and the uniqueness of the AdS soliton, Commun. Math. Phys., 241 (2003), 1-25.

[25] N. Ginoux, The Dirac Spectrum, Lecture Notes in Mathematics, 1976 (2009).

[26] C. R. Graham, J. M. Lee, Einstein metrics with prescribed conformal infinity on the ball, Adv. Math., 87 (1991), 186-225.

[27] C. Guillarmou, J. Qing, Spectral characterization of Poincaré-Einstein manifolds with infinity of positive Yamabe type, Int. Math. Res. Not., 28 (2010), 1720-1740.

[28] O. Hijazi, S. Montiel, Extrinsic Killing spinors, Math. Z., 244 (2003), $337-347$.

[29] O. Hijazi, S. Montiel, Supersymmetric rigidity of asymptotically locally hyperbolic manifolds, 2012, preprint.

[30] O. Hijazi, S. Montiel, X. Zhang, Dirac operator on embedded hypersurfaces, Math. Res. Lett, 8 (2001), 195-208.

[31] O. Hijazi, S. Montiel, X. Zhang, Conformal lower bounds for the Dirac operator of embedded hypersurfaces, Asian J. Math., 6 (2002), 23-36.

[32] N. Hitchin, Harmonic spinors, Adv. in Math., 14 (1974), 1-55.

[33] M. Kanai, On a differential equation characterizing a Riemannian structure of a manifold, Tokyo J. Math., 6 (1983), 143-151.

[34] H. B. Lawson, M. L. Michelsohn, Spin Geometry, Princeton Math. Series, vol. 38, Princeton University Press, 1989.

[35] J. M. Lee, The spectrum of an asymptotically hyperbolic Einstein manifold, Comm. Anal. Geom., 2 (1995), 253-271.

[36] B. O’Neill, Semi-Riemannian Geometry, Academic Press, 1983.

[37] J. Qing, On the rigidity for conformally compact Einstein manifolds, Intern. Math. Res. Not., 21 (2003), 1141-1153.

[38] M. Y. Wang, Parallel spinors and parallel forms, Ann. Global Anal. Geom., 7 (1989), 59-68.

[39] M. Y. Wang, On non-simply-connected manifolds with non-trivial parallel spinors, Ann. Global Anal. Geom., 13 (1995), 31-42. 
[40] X. Wang, On conformally compact Einstein manifolds, Math. Res. Lett., 8 (2001), 671-687.

[41] X. Wang, The mass of asymptotically hyperbolic manifolds, J. Diff. Geom., 57 (2001), 273-299.

[42] X. Wang, Uniqueness of the ADS space time in any dimension, Acta Math. Sin., 21 (2005), 917-922.

[43] E. Witten, S.-T. Yau, Connectedness of the boundary in the AdS/CFT correspondence, Adv. Theor. Math. Phys.,3 (1999), 1635-1655.

Institut Élie Cartan, Université de Lorraine

Nancy, B.P. 239, 54506 Vandeeuvre-Lès-Nancy Cedex, France

E-mail address: Oussama.Hijazi@univ-lorraine.fr

Departamento de Geometría y Topología

Universidad de Granada, 18071 Granada, Spain

E-mail address: smontiel@ugr.es 
